\title{
Intraoperative identification of esophageal sentinel lymph nodes with near-infrared fluorescence imaging
}

Cherie P. Parungo, $\mathrm{MD}^{\mathrm{a}}$

Shunsuke Ohnishi, MD, $\mathrm{PhD}^{\mathrm{c}}$

Sang-Wook Kim, PhD ${ }^{d}$

Sunjee Kim, $\mathrm{PhD}^{\mathrm{d}}$

Rita G. Laurence, BS ${ }^{a}$

Edward G. Soltesz, MD

Frederick Y. Chen, MD, PhD

Yolonda L. Colson, MD, PhD

Lawrence H. Cohn, MD

Moungi G. Bawendi, $\mathrm{PhD}^{\mathrm{d}}$

John V. Frangioni, MD, $\mathrm{PhD}^{\mathrm{c}}$

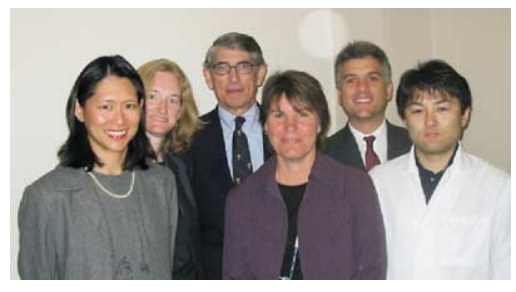

Parungo, Colson, Cohn, Laurence, Frangioni, Ohnishi (left to right)
From the Cardiac Surgery Research Laboratory $^{\mathrm{a}}$ and Department of Thoracic Surgery, ${ }^{\text {b }}$ Brigham \& Women's Hospital, and the Division of Hematology/Oncology and Department of Radiology, Beth Israel Deaconess Medical Center, ${ }^{\mathrm{C}}$ Boston, Mass, and the Department of Chemistry, Massachusetts Institute of Technology, ${ }^{\mathrm{d}}$ Cambridge, Mass.

Supported by National Institutes of Health grant NRSA F32 HL72568-01 (C.P.P), the US Department of Energy (Office of Biological and Environmental Research) grant DE-FG02-01ER63188 (J.V.F.), a Proof of Principle Award from the Center for Integration of Medicine and Innovative Technology (J.V.F), and National Institutes of Health grant R21/R33 EB-00673 (J.V.F. and M.G.B.).

Received for publication May 29, 2004; revisions received July 29, 2004; accepted for publication Aug 2, 2004.

Address for reprints: John V. Frangioni, MD, $\mathrm{PhD}$, Department of Hematology/Oncology and Department of Radiology, Beth Israel Deaconess Medical Center, 330 Brookline Ave, Room SL-B05, Boston, MA, 02215 (Email: jfrangio@bidmc.harvard.edu)

J Thorac Cardiovasc Surg 2005;129:844-50 $0022-5223 / \$ 30.00$

Copyright (C) 2005 by The American Association for Thoracic Surgery

doi:10.1016/j.jtcvs.2004.08.001
Objective: In esophageal cancer, selective removal of involved lymph nodes could improve survival and limit complications from extended lymphadenectomy. Mapping with vital blue dyes or technetium Tc-99m often fails to identify intrathoracic sentinel lymph nodes. Our purpose was to develop an intraoperative method for identifying sentinel lymph nodes of the esophagus with high-sensitivity nearinfrared fluorescence imaging.

Methods: Six Yorkshire pigs underwent thoracotomy and received submucosal, esophageal injection of quantum dots, a novel near-infrared fluorescent lymph tracer designed for retention in sentinel lymph nodes. Six additional pigs underwent thoracotomy and received submucosal esophageal injection of CW800 conjugated to human serum albumin, another novel lymph tracer designed for uptake into distant lymph nodes. Finally, 6 pigs received submucosal injection of the fluorophore-conjugated albumin with an endoscopic needle through an esophagascope. These lymph tracers fluoresce in the near-infrared, permitting visualization of migration to sentinel lymph nodes with a custom intraoperative imaging system.

Results: Injection of the near-infrared fluorescent lymph tracers into the esophagus revealed communicating lymph nodes within 5 minutes of injection. In all 6 pigs that received quantum dot injection, only a single sentinel lymph node was identified. Among pigs that received fluorophore-conjugated albumin injection, in 5 of 12 a single sentinel lymph node was revealed, but in 7 of 12 two sentinel lymph nodes were identified. There was no dominant pattern in the appearance of the sentinel lymph nodes either cranial or caudal to the injection site.

Conclusion: Near-infrared fluorescence imaging of sentinel lymph nodes is a novel and reliable intraoperative technique with the power to assist with identification and resection of esophageal sentinel lymph nodes.

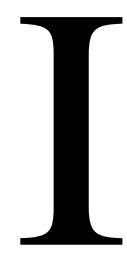

n esophageal cancer, extensive lymphadenectomy may improve survival ${ }^{1}$; however, it also increases morbidity and mortality. ${ }^{2}$ Selective removal of involved lymph nodes could minimize the extent of lymphadenectomy yet still provide accurate staging and local control. Unfortunately, current methods of identifying involved lymph nodes, including computed tomography, positron emission tomography, and endoscopic ultrasonography, are only $60 \%$ to $80 \%$ accurate. ${ }^{3}$ Similarly, sentinel lymph node (SLN) mapping with vital blue dyes or radioactive tracers is neither sufficient nor adequate in the setting of esophageal cancer. In the thorax, intraoperative mapping with blue dye identifies the SLNs in fewer than $50 \%$ of cases because of poor tissue penetration and 
anthracotic mediastinal lymph nodes. ${ }^{4}$ Similarly, handheld gamma probes miss more than $50 \%$ of radioactive nodes from the esophagus. ${ }^{5}$ The purpose of this study was to test the ability of two novel near-infrared (NIR) fluorescent lymph tracers to identify SLNs of the esophagus. Our hope was that these findings could provide the basis for advancing further studies of NIR fluorescence technology for clinical applications, namely providing selective removal of esophageal lymph nodes for accurate staging information without the need for extensive lymphadenectomy.

\section{Material and Methods \\ Preparation of Lymph Tracers}

NIR quantum dots (QDs) designed for retention in the first draining lymph node have been previously described elsewhere. ${ }^{6}$ Briefly, these type II core/shell QDs are semiconductor nanocrystals that contain an inorganic core of cadmium telluride, an inorganic shell of cadmium selenide, and an outer organic coating of solubilizing oligomeric phosphines. Hydrodynamic diameter measured by gel filtration is 15 to $20 \mathrm{~nm}$. These particular QDs were engineered to fluoresce in the NIR, with peak emission at $840 \mathrm{~nm}$. A stock solution of $0.2-\mu \mathrm{mol} / \mathrm{L}$ QDs in phosphate-buffered saline solution, $\mathrm{pH}$ 7.4, was used for all studies.

HSA800, designed with a hydrodynamic diameter of $7 \mathrm{~nm}$ for migration to distant lymph nodes, will be described in detail elsewhere (Ohnishi et al, unpublished data). Briefly, human serum albumin (HSA) was covalently conjugated to the NIR fluorophore CW800 by means of an amide bond (HSA800). The ratio of CW800 to albumin was 3.4:1. Peak absorbance and emission of HSA800 were $778 \mathrm{~nm}$ and $795 \mathrm{~nm}$, respectively, in phosphate buffered saline solution, $\mathrm{pH} 7.4$. A stock solution of $0.8-\mathrm{mg} / \mathrm{mL}$ HSA800 in phosphate-buffered saline solution was used for all studies. These fluorescent biologic labels are excellent tracers for SLN mapping because they are highly fluorescent, nonradioactive, and easily visible deep within tissue.

\section{Direct Injection of QDs into the Submucosa of the Esophagus}

All protocols were in compliance with the "Guide for the Care and Use of Laboratory Animals" (http://www.nap.edu/catalog/ $5140 . \mathrm{html}$ ). Adult, male Yorkshire pigs (mean weight $35 \mathrm{~kg}$ ) were anesthetized intramuscularly with tiletamine and zolazepam (Telazol, $4.4 \mathrm{mg} / \mathrm{kg}$ ) and xylazine $(2.2 \mathrm{mg} / \mathrm{kg})$ and subsequently with isoflurane ( $0.5 \%-5 \%$ to effect). The animals were intubated, prepared, and draped in the usual fashion. Pigs underwent a left cervical, right thoracotomy, or left subcostal incision to visualize the upper, middle, or lower esophagus, respectively $(\mathrm{n}=2$ for each group). Submucosal injection of $100 \mu \mathrm{L}$ of $0.2-\mu \mathrm{mol} / \mathrm{L}$ NIR QDs in phosphate-buffered saline solution, $\mathrm{pH}$ 7.4, into either the upper, middle, or lower esophagus was performed. Injections of the lymph tracer were performed by the same surgeon with identical technique.

\section{Direct or Endoscopic Injection of HSA800}

Six pigs underwent the same procedure except with direct injection of HSA800 (dye concentration $10 \mu \mathrm{mol} / \mathrm{L}$ ) into the submucosa of the upper, middle, or lower esophagus ( $\mathrm{n}=2$ for each segment).
An additional 6 pigs underwent submucosal injection of $100 \mu \mathrm{L}$ HSA800 with a rigid esophagascope and an endoscopic injecting needle. Injections of the lymph tracer were performed by the same surgeon with identical technique.

\section{Identification and Resection of the SLNs}

The NIR-fluorescence imaging system has been described elsewhere in detail. ${ }^{7}$ Briefly, it is composed of a $400-$ to $700-\mathrm{nm}$ "white" light $\left(0.5 \mathrm{~mW} / \mathrm{cm}^{2}\right)$ and a 725 - to $775-\mathrm{nm}$ NIR fluorescence excitation light $\left(5 \mathrm{~mW} / \mathrm{cm}^{2}\right)$. Images of white light and NIR fluorescence can be displayed separately and merged. The entire apparatus is suspended on an articulated arm over the surgical field, thus permitting noninvasive and nonintrusive imaging. Realtime video images, refreshed 15 times per second, and zooming capability allowed precise localization and dissection as needed.

The migration of the lymph tracers from the injection site either cranially or caudally was imaged in real time. Selected pigs were imaged 4 hours after injection of QDs and HSA800 to detect new fluorescence or loss of fluorescence in lymph nodes of the esophagus. SLNs and control nonfluorescing lymph nodes were dissected under NIR-fluorescence image guidance, embedded with Tissue-Tek OCT compound (Fisher Scientific, Pittsburgh, Pa), frozen in liquid nitrogen, and sectioned at $6 \mu \mathrm{m}$. Alternate cuts were examined by hematoxylin and eosin staining and NIR fluorescence imaging. The NIR fluorescence was visualized through a modified microscope with custom-designed optics, as described previously elsewhere. ${ }^{8}$

\section{Statistical Analysis}

Associations between categorical parameters were assessed with Fisher exact and paired Student $t$ tests.

\section{Results}

\section{Direct Injection of QDs into the Esophagus}

Intrinsic autofluorescence of the esophagus and surrounding thoracic tissue was minimal (Figure 1). Direct injection of QDs into the esophageal wall and QD migration to a single SLN was visible in real time. A single SLN was identified within 5 minutes in $100 \%$ of pigs (Figure 1). SLN did not diminish in fluorescence, nor did additional SLNs appear after 4 hours (data not shown).

\section{Direct and Endoscopic Injection of HSA800 into the Esophagus}

Direct and endoscopic injection of HSA800 produced comparative results of bright fluorescence tracer in the wall of the esophagus against a low background (Figure 2). In 12 pigs receiving HSA800, the mean time of SLN visualization was 3 minutes, with a range of 30 seconds to 5 minutes (Figure 3, A ). As with QDs, fluorescence within SLNs did not diminish, and no additional lymph nodes were found after 4 hours (Figure 3). One sentinel lymph node was identified in 5 of the 12 pigs. Two nodes were identified in 7 of the 12 pigs. More specifically, one lymphatic directed the HSA800 to two different nodes (Figure 3, $B$ ). In 1 case, the HSA800 entered a node through an efferent lymphatic 
Color
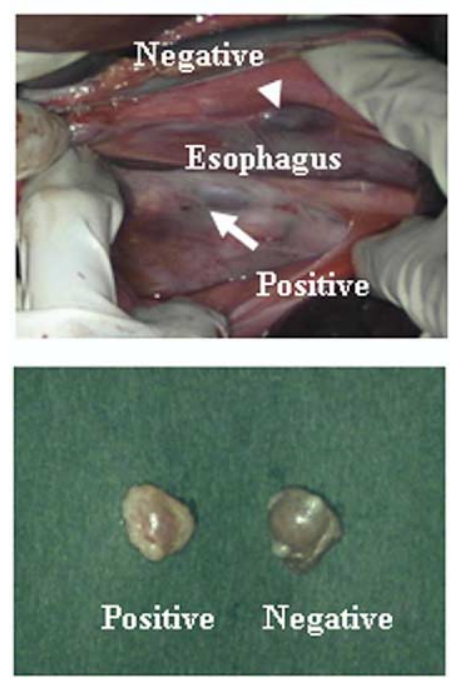

NIR Fluorescence
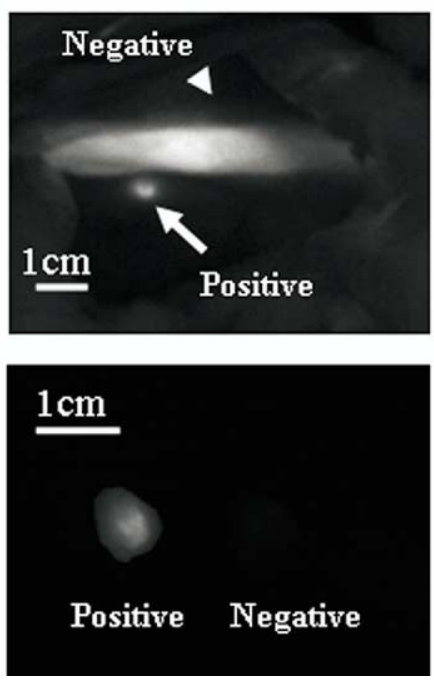

\section{Color-NIR Merge}
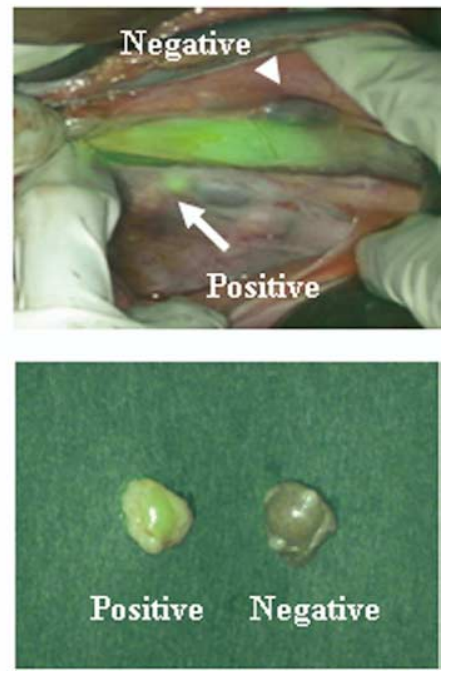

Figure 1. QDs migrate from esophagus to specific lymph nodes of pig. Top row shows in vivo esophagus of pig visualized with color video (left column), NIR fluorescence (middle column), and color-NIR merge images (right column). Lymph nodes shown are positive (arrows) and negative (arrowheads) for OD uptake. Bottom row shows same lymph nodes after resection.

TABLE 1. Distribution of the SLNs either cranial or caudal to the injection site

\begin{tabular}{lcc}
\hline & $\begin{array}{c}\text { SLNs cranial to } \\
\text { injection site }\end{array}$ & $\begin{array}{c}\text { SLNs caudal to } \\
\text { injection site }\end{array}$ \\
\hline $\begin{array}{l}\text { Injection location } \\
\text { Proximal esophagus }\end{array}$ & 1 & 5 \\
$\quad$ Mid esophagus & 4 & 2 \\
$\quad$ Distal esophagus & 3 & 3 \\
Tracer & 3 & \\
ODs & 5 & 3 \\
HSA800 & & 7 \\
Method of injection & 6 & 6 \\
Direct injection & 2 & 4 \\
$\quad$ Endoscopic injection & 8 & 10 \\
Total & & \\
\hline
\end{tabular}

Data represent numbers of animals. There was no predictable pattern of location of the SLNs regardless of location of injection, NIR fluorescent tracer used, or method of injection (direct or endoscopic).

vessel and stayed within the node but also continued out of the node through an afferent lymphatic vessel in a matter of seconds (Figure 3, $C$ ).

\section{Distribution of SLNs of the Esophagus}

There was no predictable distribution of the SLNs in relation to the injection site (Table 1). Among the 18 pigs receiving NIR fluorescent tracer injection, 8 had a SLN cranial to the injection site, and 10 had a SLN caudal to the injection site. There was no difference in the distribution of the SLN when using QDs versus HSA800 $(P=.50)$ or when using direct injection versus endoscopic injection $(P=.70)$.

\section{Histologic Results}

Hematoxylin and eosin images confirmed that brightlyfluorescent specimens were lymph nodes. NIR images confirmed that both QDs and HSA800 could be detected within the substructure of the lymph node (Figure 4). HSA800 fluorescence was more robust than QD fluorescence in histologic samples. As a control, nonfluorescing lymph nodes were also sectioned and imaged. As expected, these nodes showed no fluorescence under the NIR-fluorescent microscope (Figure 4).

\section{Discussion}

In esophageal cancer, aggressive lymphadenectomy may remove tissue at risk of involvement, however, this comes at the expense of higher morbidity and mortality. An intraoperative SLN evaluation could help direct the extent of lymphadenectomy and thus improve staging and treatment on a patient-specific basis. ${ }^{9}$ Although the concept of SLNs can be applied to esophageal cancer, ${ }^{10}$ there are several disadvantages to using technetium Tc $99 \mathrm{~m}$ and vital blue dyes. Technetium Tc $99 \mathrm{~m}$ colloids require radioactive protocols and storage. The handheld gamma counter not only is cumbersome but fails to detect more than $50 \%$ of radioactive esophageal nodes subsequently identified after resection. ${ }^{5}$ With technetium Tc $99 \mathrm{~m}$, radioactive signal from the 

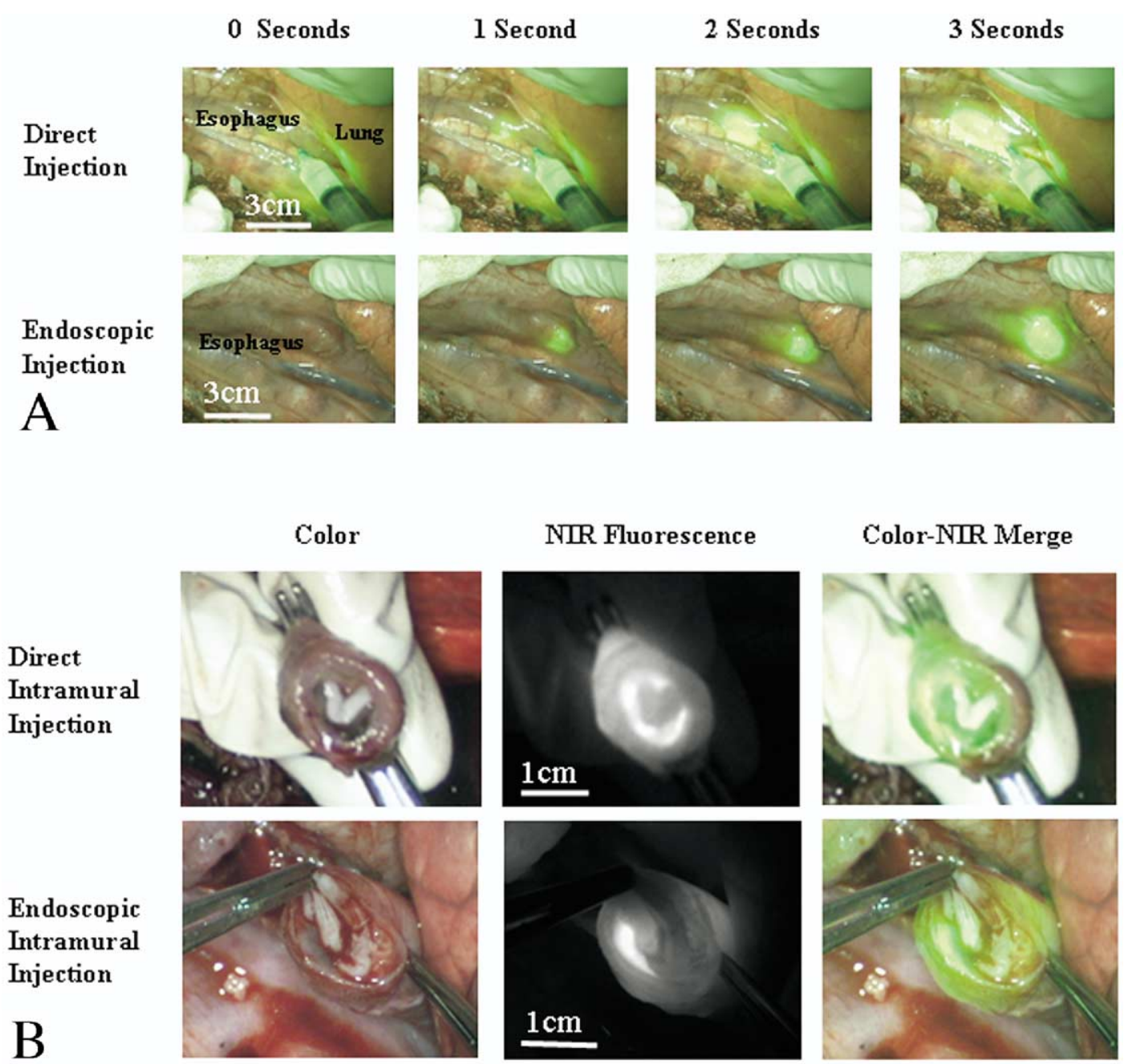

Figure 2. In vivo imaging of HSA800 injection into esophagus of pig. A, HSA800 directly injected into esophagus as visualized with color-NIR merge imaging (top row) and HSA800 endoscopically injected into esophagus as visualized with color-NIR merge imaging (bottom row). B, Cross-section of injection site showing intramural location of HSA800 in esophagus, visualized with color video (left column), NIR fluorescence (middle column), and color-NIR merge images (right column). Top row shows cross-section from direct intramural injection; bottom row shows cross section from endoscopic intramural injection.

esophageal injection site interferes with detection of SLNs, also known as "shine-through" effect. Vital blue dyes have limited application in detection of thoracic lymph nodes because of the presence of innate lymph node anthracosis. There is also increasing concern regarding anaphylactic reactions to vital blue dyes. ${ }^{11}$

There are several advantages of NIR fluorescence technology for SLN identification in the esophagus. Both QDs and HSA800 are powerful lymph tracers that fluoresce brightly against a low background and distinctly from the injection site. These NIR-fluorescent lymph tracers do not leak from tissues, nor do they stain the field of dissection. Communicating lymph nodes can be seen 5 minutes to 4 hours later, thus providing great flexibility of preoperative or intraoperative administration. With NIR-fluorescent imaging, the entire surgical field can be imaged in real-time for in situ SLNs, thus minimizing sampling error. NIR fluorescence imaging can provide magnified images to assist with precise and complete resection of the SLN without having to handle another instrument, such as a gamma probe. NIR-fluorescing tracers withstand histologic processing, thus providing another degree of certainty in the identification of SLNs.

SLN mapping with NIR technology could pose some of the same challenges as SLN mapping with technetium Tc $99 \mathrm{~m}$ or vital blue dyes. Tumor within a lymph node could potentially alter lymphatic flow, deny access of lymph tracer to a positive node, and possibly create a false-negative SLN. 

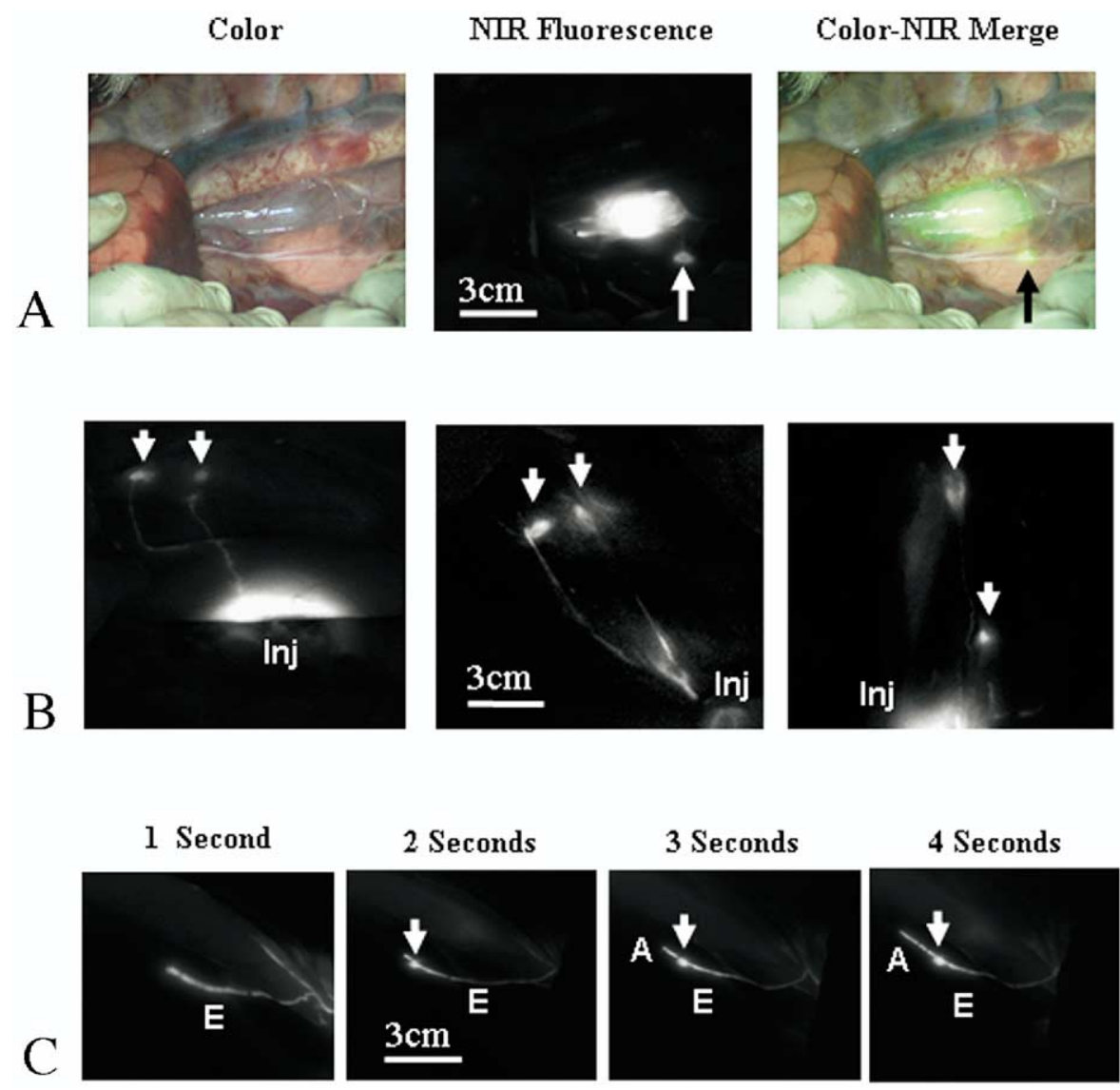

Figure 3. HSA800 migrates from esophagus to specific lymph nodes of pig. A, Top row shows in vivo pig esophagus visualized with color video (left), NIR fluorescence (middle), and color-NIR merge images (right). Arrows mark HSA800-positive lymph node. B, Three examples of two HSA800-positive lymph nodes (arrows) being imaged from single injection site into pig esophagus (Inj). C, Example of efferent lymphatic (E) leading to HSA800-positive lymph node (arrow) and afferent lymphatic (A) leaving same lymph node, as seen with NIR fluorescence imaging. NIR fluorescence images have identical exposure times and normalizations.

However, studies with the breast have found that tumor burden does not increase the false-negative rate. ${ }^{12}$ Tumor burden can decrease the success of finding SLNs. Therefore failure to identify a SLN or suspicious nodes found at the time of SLN biopsy warrants complete lymphadenectomy. These studies and practices developed for breast cancer will have to be explored in esophageal cancer.

\section{NIR-Fluorescent Lymph Tracers}

The optimal size of lymph tracers is controversial but thought to be in the range of 4 to $100 \mathrm{~nm} .^{13,14}$ Because esophageal cancer appears to metastasize to distant lymph nodes, suggesting a broad distribution of drainage or skip metastasis, ${ }^{15}$ a small, mobile particle such as HSA800 may be an ideal tracer for SLN mapping. QDs, with a larger diameter, migrated only to the first draining lymph node. In contrast, HSA800 could be found in two draining nodes in 7 of 12 pigs. In 1 case, HSA800 identified a lymph node and also partially traveled out to identify a second lymph node. Because HSA800 is smaller than QDs, it may have access to smaller lymphatics that QDs cannot traverse. The clinical significance of a second SLN in the esophagus will have to be determined with future studies. However, the presence of multiple communicating nodes and even multiple lymphatic patterns does not preclude the application of accurate SLN identification in the esophagus. Multiple SLNs are phenomenon accepted in SLN mapping of breast and melanoma. ${ }^{16}$ In fact, the identification of multiple SLNs improved the false-negative rate to $4.3 \%$ from $14.3 \%$ when only one SLN was identified. ${ }^{17}$ The ability of HSA800 to identify multiple nodes may make it a better lymph tracer to limit falsenegative results; however, specific studies directed at this question will have to be performed.

A potential limitation of QDs is their unknown toxicity. The individual metals comprising the inorganic core and shell have known toxic effects, especially at concentrations 
$\mathrm{H} \& \mathrm{E}$

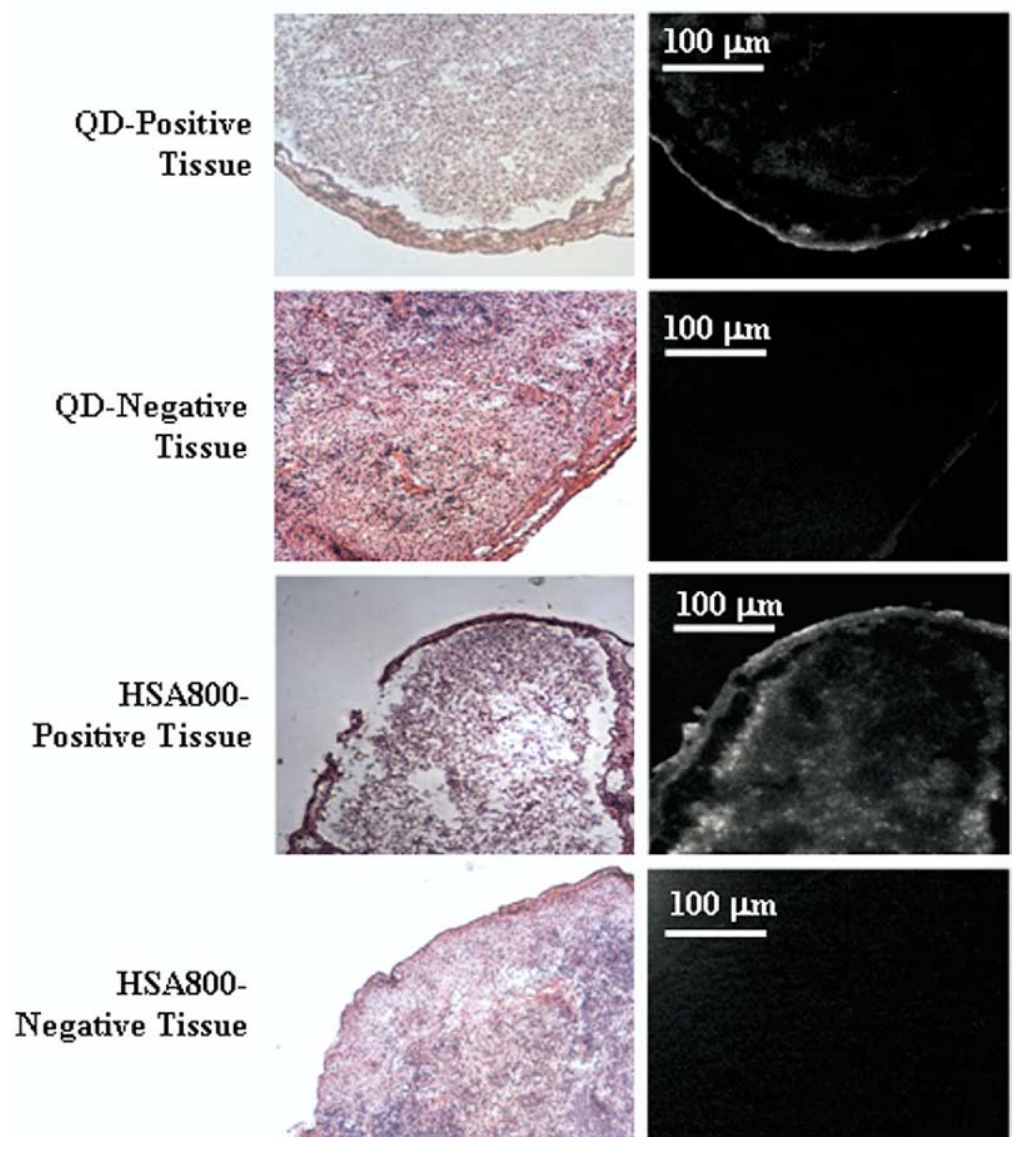

Figure 4. Histologic photomicrographs of NIR-fluorescent positive and negative tissue. Top row shows QD-positive lymph node of pig with hematoxylin and eosin (H\&E) staining (left) and consecutive unstained section of same node imaged for NIR fluorescence (right). Second row shows QD-negative lymph node of pig. Third row shows HSA800-positive lymph node of pig. Bottom row shows HSA800-negative lymph node of pig. Corresponding positive and negative NIR fluorescence images have identical exposure times and normalizations. (Original magnification $100 \times$.

higher than those used in our study. The toxicity of these metals when complexed as salts in the core of a QD is unknown. In our studies, there were no signs of acute toxicity, namely changes in heart rate, rhythm, blood pressure, or oxygen saturation. HSA800 has greater potential for immediate clinical application because it is purely organic and is the product of nontoxic components. However, studies directed at establishing the toxicity, if any, of these tracers must still be performed before applying the technology to human patients.

Last, our data corroborate earlier studies showing an unpredictable drainage pattern of the esophagus. ${ }^{18}$ Injections of the lymph tracers, whether direct or endoscopic, were performed by the same surgeon with identical technique. Yet, regardless of tracer size, mode of injection, or location of injection, there was no consistent pattern of drainage from injection sites. These findings emphasize the need for a reliable, sensitive, patient-specific method for identifying SLNs of the esophagus. NIR-fluorescence imaging is not only a promising method for identifying SLNs but also a powerful instrument for image-guided dissection.

Advancing this technology to SLN biopsy in human beings could have clinical impact on both staging and treatment in esophageal cancer. Selective removal of the SLNs could provide staging information without the morbidity and mortality of routine extensive lymphadenectomy. If the SLN proved positive, aggressive treatment options such as extended lymphadenectomy or more aggressive adjuvant therapy could be appropriately offered. In conclusion, our studies show the feasibility of patient-specific identification of SLNs of the esophagus with NIR fluorescence technology. However, further studies are needed to apply this technique in humans. 


\section{References}

1. Altorki NK, Skinner DB. Occult cervical nodal metastasis in esophageal cancer: preliminary series of the three-field lymphadenectomy. J Thorac Cardiovasc Surg. 1997;113:540-4.

2. Altorki N, Kent M, Ferrara C, Port J. Three-field lymph node dissection for squamous cell and adenocarcinoma of the esophagus. Ann Surg. 2002;236:177-83.

3. Romagnuolo J, Scott J, Hawes RH, Hoffman BJ, Reed CE, Aithal GP, et al. Helical CT versus EUS with fine needle aspiration for celiac nodal assessment in patients with esophageal cancer. Gastrointest Endosc. 2002;55:648-54.

4. Little AG, DeHoyos A, Kirgan DM, et al. Intraoperative lymphatic mapping for non-small cell lung cancer: the sentinel node technique. J Thorac Cardiovasc Surg. 1999;117:220-4.

5. Yasuda S, Shimada H, Chino O, Tanaka H, Kenmochi T, Takechi M, et al. Sentinel lymph node detection with Tc-99m tin colloids in patients with esophagogastric cancer. Jpn J Clin Oncol. 2003;33:68-72.

6. Kim S, Lim YT, Soltesz EG, De Grand AM, Lee J, Nakayama A, et al. Near-infrared fluorescent type II quantum dots for sentinel lymph node mapping. Nat Biotechnol. 2003;22:93-7.

7. De Grand AM, Frangioni JV. An operational near-infrared fluorescence imaging system prototype for large animal surgery. Technol Cancer Res Treat. 2003;2:553-62.

8. Zaheer A, Lenkinski RE, Mahmood A, Jones AG, Cantley LC, Frangioni JV. In vivo near-infrared fluorescence imaging of osteoblastic activity. Nat Biotechnol. 2001;19:1148-54.

9. Matsubara T, Ueda M, Kaisaki S, Kuroda J, Uchida C, Kokudo N, et al. Localization of initial lymph node metastasis from carcinoma of the thoracic esophagus. Cancer. 2000;89:1869-73.
10. Kato H, Miyazaki T, Nakajima M, Takita J, Sohda M, Fukai Y, et al. Sentinel lymph nodes with technetium-99m colloidal rhenium sulfide in patients with esophageal carcinoma. Cancer. 2003;5:932-9.

11. Sprung J, Tully M, Ziser A. Anaphylactic reactions to isosulfan blue dye during sentinel node lymphadenectomy for breast cancer. Anesth Analg. 2003;96:1051-3.

12. Wong SL, Edwards MJ, Chao C, Simpson D, McMasters KM. The effect of lymphatic tumor burden on sentinel lymph node biopsy results. Breast J. 2002;8:192-8.

13. Noguchi N. Sentinel lymph node biopsy and beast cancer. Br J Surg. 2002;89:21-34.

14. Watanabe T, Kimijima I, Ohtake T, Tsuchiya A, Shishido F, Takenoshida S. Sentinel node biopsy with technetium-99m colloidal rhenium sulphide in patients with breast cancer. Br J Surg. 2001;88: 704-7.

15. Nomura T, Onda M, Miyashita M, Makino H, Maruyama H, Nagasawa $\mathrm{S}$, et al. Wide-spread distribution of sentinel lymph nodes in esophageal cancer. J Nippon Med Sch. 2001;68:393-6.

16. McMasters KM, Reintgen DS, Ross MI, Wong SL, Gershenwald JE, Krag DN, et al. Sentinel lymph node biopsy for melanoma: how many radioactive nodes should be removed? Ann Surg Oncol. 2001;8:192-7.

17. Wong SL, Edwards MJ, Chao C, Tuttle TM, Noyes RD, Carlson DJ, et al. Sentinel lymph node biopsy for breast cancer: impact of the number of sentinel nodes removed on the false-negative rate. J Am Coll Surg. 2001;192:684-91.

18. Hosch SB, Stoecklein NH, Pichlmeier U, Rehders A, Scheunemann P, Niendorf A, et al. Esophageal cancer: the mode of lymphatic tumor cell spread and its prognostic significance. J Clin Oncol. 2001;19: $1970-5$. 